\title{
Androgen Insensitivity Syndrome (AIS): A Case History
}

Khatun Hazera ${ }^{1}$, Rahman Tarannum ${ }^{2}$

\begin{abstract}
Androgen Insensitivity Syndrome (AIS) is an inherited $X$-linked recessive disease with a mutation in the Androgen Receptor (AR) gene resulting in - functioning of $Y$ sex chromosome and abnormality on $X$ sex chromosome, where affected individuals have male chromosomes and male gonads with complete or partial feminization of the external genitals. Recently a case of complete Androgen Insensitivity Syndrome (CAIS) is reported at Shaheed Suhrawardy Medical College, Dhaka. A 15 years old school girl was admitted with primary amenorrhea with bilateral inguinal hernia. Diagnosis of complete AIS is confirmed by discovering an adult male testosterone level, 46, XY karyotype, absence of axillary and pubic hair and a shallow vagina, with no cervix or uterus.
\end{abstract}

Key Words: Androgen Insensitivity Syndrome (AIS), 46 XY karyotype.

\section{INTRODUCTION}

Androgen insensitivity syndrome (AIS) is a disorders of sexual differentiation that results from mutations of the gene encoding the androgen receptor. ${ }^{1} \mathrm{~A}$ woman with complete androgen insensitivity syndrome (CAIS) has a female external appearance despite a 46XY karyotype and undescended testes, this condition termed testicular feminization in the past. ${ }^{2}$ It was first reported in 1817 by Steglehner, he described about normal women with undescended testis. In 1957 Wilkins found tissue unresponsive to androgen. ${ }^{3}$

Androgen receptor gene cloned and sequenced in 1988. Major changes in the understanding and management of the various forms of AIS have occurred since 1990. More importantly, patient advocacy groups for AIS and other intersex conditions have increased public awareness of these disorders to understanding of gender identity, emphasized the value of accurate and sophisticated information for patients and physicians. The initial complaints may be primary amenorrhoea and/or inguinal swelling in spite of normal body development (except absence of axillary and pubic hair). Diagnosis of complete AIS is confirmed by discovering an adult male testosterone level, 46XY karyotype, absence of axillary and pubic hair and a shallow vagina, with no cervix or uterus.

The disease affects 1 in 20,400 people; of them $2 / 3$ rd of the cases are inherited from mother and $1 / 3$ of cases come from a spontaneous mutation in the egg. For a carrier woman, there is 1 in 4 chance in each pregnancy that the child will have AIS and in 2 chances if the fetus is male. The disease has no racial differences and no effects on life expectancy.

\section{Case Reports:}

A 15 years old school girl, hailing from Valuka, Mymensingh has got admitted in Shaheed Suhrawardy Medical College \& Hospital, Dhaka, with the complaints of non-establishment of menstruation and bilateral inguinal swelling for 1 year. She has no history of cyclical lower abdominal pain, weight loss, visual disturbance, severe headache or chronic cough.

\section{Authors'Information:}

1. Dr. Hazera Khatun, MBBS, MCPS, MS (Obstetrics and Gynaecology), Associate Professor (Obstetrics and Gynaecology), Shaheed Suhrawardy Medical College, Dhaka.

2. Dr. Tarannum Rahman, MBBS (Shaheed Suhrawardy Medical College), MPH (JPGSPH, BRACU, Dhaka), Clinical Co-ordinator, United Hospital, Dhaka.

Address of Correspondance: Dr. Hazera Khatun, MBBS, MCPS, MS (Obstetrics and Gynaecology), Associate Professor (Obstetrics and Gynaecology), Shaheed Suhrawardy Medical College, Dhaka. Cell: +8801711529768, Email: khatunhazera73@gmail.com 
On examination she was attractive, feminine looking, height 5'4" and weight $52 \mathrm{~kg}$. Her skin was smooth and hair less, axillary and pubic hair - absent, normal female voice, breast welldeveloped and its development started at 11 years of age. She was not anemic, thyroid gland not enlarged and lymph nodes - not palpable. Per abdominal examination revealed bilateral inguinal swelling situated $1 \mathrm{~cm}$ above and lateral to the pubic tubercle on standing position and reduced spontaneously on supine position. These swellings were about $2.5 \times 2 \mathrm{~cm}$ oval, non-tender and firm in consistency, smooth and reducible. Per vaginal examination revealed, vulva and perineum - normal, vagina admits one finger, length about $4 \mathrm{~cm}$ and blind upper end. Cervix and uterus could not be felt. Digital rectal examination excluded presence of uterus.

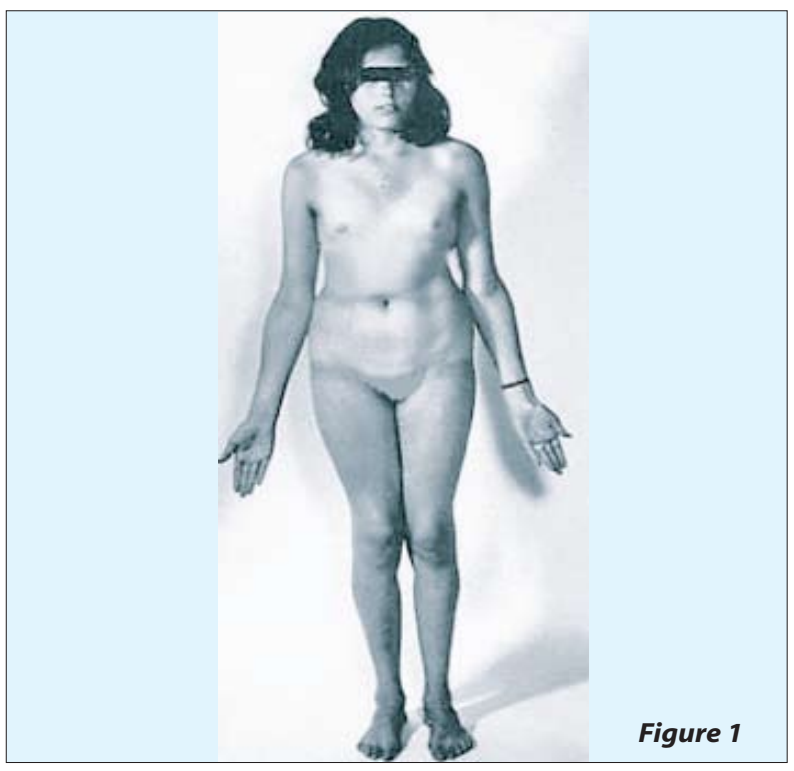

FIGURE 1: A women with an XY chromosome pattern but insensitivity to androgens. Complete Breast development, but absence of pubic hair.

USG of whole abdomen showed cytopic gonads with absence of uterus, Karyotyping - 46 XY. Hormone assay revealed S. Testosterone 17.6 $\mathrm{nmol} / \mathrm{L}$, S. Oestrogen $48.05 \mathrm{pg} / \mathrm{ml}$, S. FSH 7.01 $\mathrm{mIU} / \mathrm{ml}$. Her blood count, blood glucose, chest $X-$ ray, ECG, thyroid hormone assay and urine analysis and culture, all were within normal limits.

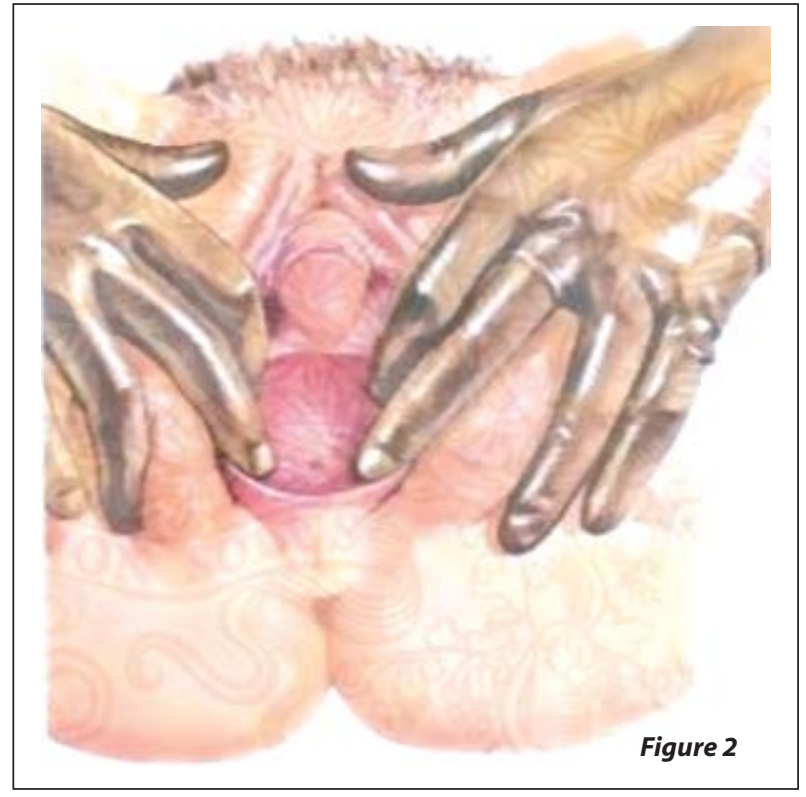

FIGURE 2: Enlarged Clitoris and absence of uterus

After analyzing all investigation reports and examination of the patient she was diagnosed as a case of CAIS. Within a sort span of her diagnosis bilateral Orchidectomy was done. Histopathological examination showed testicular tissue with atrophy of seminiferous tubules without any evidence of spermatogenesis, one specimen attached with tubular structure is compatible with dilated spermatic cord. ${ }^{4}$ Her postoperative period was uneventful and she was discharged at her $14^{\text {th }}$ day of operation with necessary advice.

\section{Discussion}

Androgen Insensitivity Syndrome, formerly known as testicular feminization syndrome, is an inherited $X$-linked recessive disease with a mutation in the Androgen Receptor (AR) gene resulting in functioning of $Y$ sex chromosome and abnormality on $X$ sex chromosome, where affected people have male chromosomes and male gonad with complete or partial feminization of the external genitals. ${ }^{5}$ This failure of virilization can be either CAIS or partial Androgen Insensitivity Syndrome (PAIS), depending on the amount of residual receptor function. ${ }^{5}$ 
Both individuals with PAIS and with CAIS have 46, XY karyotypes. The phenotypes of individuals with CAIS have female external genitalia with normal labia, clitoris and vaginal introitus. ${ }^{6}$ The phenotype of individuals with PAIS may range from mildly virilized female external genitalia (cliteromegaly without other external anomalies), reifenstein syndrome, infertile male syndrome; Undervirilized fertile male syndrome to $X$ linked spinal and bulbar muscular atrophy.

Either case (CAIS \& PAIS), affected individuals have normal testis with normal production of testosterone and normal conversion to dihydrotestoterone (DHT) which differentiate this condition from 5-a reductase deficiency. The testis produce Mullerian inhibitory factor, so affected individual do not have fallopian tubes, uterus or proximal (upper) vagina. Internationality AIS incidence of approximately 1 case per 20,400 live born males. That included only hospitalized cases, so the true incidence of AIS may be higher. CAIS appears common than PAIS and no racial difference in incidence. ${ }^{7}$

Androgen Insensitivity Syndrome is the little medical morbidity or mortality. Over time, untreated patients have a risk of malignant degeneration and development of gonadoblastoma of the testis. The patients with CAIS and PAIS retaining their testis after puberty have a $25 \%$ chance of developing benign tumors and a $4-9 \%$ chance of malignancy. The tumor is considered cured without need for further therapy if it is removed while still limited to the interior of the testis capsule. The tumor is considered curable in most patients even when undetected at this early state. ${ }^{8}$

The basic etiology of AIS is a loss of function mutation in the AR gene. This AR gene has been localized to the long arm of the $X$ chromosome (i.e. Xq 11-13). Over 200 such mutations have been described, including complete or partial gene deletions, point mutations, and small insertion/deletions. These mutations can cause a variety of functional defects, ranging from a complete loss of receptor on the cell surface because of incomplete protein synthesis to decrease substance binding affinity. Altered substrate binding affinity can cause a signal transmission loss, despite normal cell surface receptor numbers. The AIS gives rise to ambiguity between the genetic and the phenotypic gender, a person with AIS is a $46, \mathrm{XY}$ but a phenotypic female.

Most cases of CAIS are identified in the newborn period by the presence of inguinal masses, which later are identified as testis during surgery. Childhood growth is normal and they may also present with unilateral or bilateral inguinal lump that are identified as testis during surgery. Puberty tends to begin slightly later than the average for girl. Adolescent patients, notable findings primary amenorrhoea with or without inguinal masses. In addition patients have no pubic and feminine look, tall, normal female voice and breast is well developed as a result of conversion of testosterone to estrogen, non functioning testosterone and DHT. Patients with PAIS may have a highly variable genital appearance. Adolescents may have pubic hair, although usually less than normal and may have progressive clitoral enlargement and other signs of masculinization. The differentials of AIS are Mayer-Rokitansky Syndrome (Mullerian agenesis), 5-a reductase deficiency Testicular Dysgenesis.

Androgen Insensitivity Syndrome (CAIS \& PAIS) is diagnosed during pregnancy by chorionic villus sampling (9-12 weeks), Ultrasonogram and amniocentesis (after 16 weeks). After birth, Karyotyping of the individual-shows 46, XY, Pelvic USG examinations reveals absence of Mullerian structure (uterus, fallopian tubes, upper part of vagina). Hormone measurements in pubertal age with individuals of CAIS are characterized by total testosterone levels in the upper or normal male range rather than female range, estradiols levels mildly elevated or normal female range, mildly elevated LH levels, normal FSH levels. Mutation analysis of androgen receptor gene is now commercially available in many countries. It detects $95 \%$ of mutations. Histology of the testis in patients with CAIS or PAIS should show fairly normal testicular structure with numbers of spermatozoid and/or sperm may be reduced markedly in post pubertal patients. 
Accurate and sensitive explanations of the facts should be conveyed to the patients and families in an age appropriate manner. Counseling should be done as described in published recommendations for CAIS management. Many women with CAIS find value in making connections with others similarly affected. The internet now provides the simplest method of connecting with such support group (AIS Support Group, AISSG). As a part of genetic counseling, when a woman is diagnosed with CAIS or PAIS, referral to a genetic counselor may be warranted to explain the implications of the X-linked recessive inheritance. The mother of the woman with AIS is likely to be an unaffected carrier of the gene on one of her $\mathrm{X}$ chromosomes.

A mother who carries the defect will, on average, pass it to $50 \%$ of her children, whether $X X$ of $X Y$. Those who are $X X$ will be similarly unaffected carriers who can pass it to succeeding generations. Those who are $X Y$ will have the condition but, being infertile, cannot pass it. If the family is large, other members can be found who have or carry AIS. Many women with AIS will be able to identify affected maternal relatives such as aunts or great aunts. Carrier detection by gene testing is now possible. A small percentage of new cases of AIS are due to new, spontaneous mutations, and the above information about the family is not applicable. ${ }^{9}$

Surgical care (Orchidectomy) during the late teenage or early 20 s has been preferred, but Optimal timing of removal of the testes has been the management issue, most often debated by physicians, though whether it is necessary has been questioned as well. After gonadectomy patients may lose their libido and estrogen production from testosterone might be stopped. ${ }^{9}$ Hormone replacement is indicated when testes have been removed, estrogen needs to be given in order to support pubertal development, bone development and completion of growth. Among estrogen preparations available, transferal patches are gaining in popularity. Since there is no uterus, progesterone is not considered necessary. Women for whom vaginal shallowness is a problem, enlargement can be achieved by a prolonged course of self-dilation. Genital plastic surgery sometimes performed for adults such as vaginoplasty, phalloplasty or reconstructive surgery on the female genitalia if musculinization occur. $^{8}$

\section{Conclusion}

The study concludes that individuals with PAIS and with CAIS have 46, XY karyotypes. While the phenotypes of individuals with CAIS have female external genitalia with normal labia, clitoris and vaginal introitus, the phenotype of individuals with PAIS may range from mildly virilized female external genitalia (cliteromegaly without other external anomalies), reifenstein syndrome, infertile male syndrome; undervirilized fertile male syndrome to $X$ linked spinal and bulbar muscular atrophy. In either case, affected individuals will have normal testis with normal production of testosterone and normal conversion to DHT which differentiate this condition from 5-a reductase deficiency. The testis produce Mullerian inhibitory factor, so affected individual do not have fallopian tubes, uterus or proximal (upper) vagina.

\section{REFERENCES}

1. Gottlieb B, Beitel LK, Trifiro MA: Androgen insensitivity syndrome [Monograph online]. In: Pagon RA, Adam $\mathrm{MP}$, Ardinger $\mathrm{HH}$, et al., editors. New York: University of Washington, Seattle; 2014. [cited 2015 Aug 01; Last Update: 2014 July 10].

Available from: NCBI Bookshelf, http://www.ncbi.nlm.nih.gov/books/NBK1429

2. Brinkmann AO. Molecular basis of androgen insensitivity. Molecular and Cellular Endocrinology 2001;179(1): 105-9.

3. Hughes IA, Davies JD, Bunch TI, Pasterski V, Mastroyannopoulou K, MacDougall J. Androgen insensitivity syndrome. The Lancet 2012;380(9851): 1419-28.

4. Lubahn DB, Brown TR, Simental JA, Higgs HN, Migeon CJ, Wilson EM, French FS. Sequence of the intron/exon junctions of the coding region of the human androgen receptor gene and identification of a point mutation in a family with complete androgen insensitivity. Proceedings of the National Academy of Sciences $1989 ; 86(23): 9534-38$. 
5. Hines M, Ahmed SF, Hughes IA. Psychological outcomes and gender-related development in complete androgen insensitivity syndrome. Archives of Sexual Behavior 2003;32(2):93-101.

6. Ahmed SF, Cheng A, Dovey L, Hawkins JR, Martin H, Rowland J, Hughes IA. Phenotypic features, androgen receptor binding, and mutational analysis in 278 clinical cases reported as androgen insensitivity syndrome. J Clin Endocrinol Metab 2000;5(2):658-65.

7. Keenan BS, Meyer WJ, Hadjian AJ, Jones HW, Migeon CJ. Syndrome of Androgen Insensitivity in Man: Absence of 5 ?-Dihydrotestosterone Binding Protein in Skin Fibroblasts. J Clin Endocrinol Metab 1974;38(6): 1143-46.
8. Money J, Schwartz M, Lewis VG. Adult herotosexual status and fetal hormonal masculinization and demasculinization: 46, XX congenital virilizing adrenal hyperplasia and 46, XY androgen-insensitivity syndrome compared. Psychoneuroendocrinology 1984;9(4):405-14.

9. Wisniewski AB, Migeon $\mathrm{CJ}$, Meyer-Bahlburg HF, Gearhart JP, Berkovitz GD, Brown TR et al. Complete androgen insensitivity syndrome: long-term medical, surgical, and psychosexual outcome. J Clin Endocrinol Metab 2000;85(8):2664-69. 